\title{
Murraya paniculata (L.) Jack: A Potential Plant for Treatment of Toothache
}

\author{
Md Faisal, Md MH. Sarker, Ashif Rahman, Ahamed I. Hossain, Shahnaz Rahman, ABM Anwarul \\ Bashar, Rownak Jahan, and Mohammed Rahmatullah* \\ Faculty of Life Sciences, University of Development Alternative, Dhanmondi, Dhaka-1209, Bangladesh
}

Received: September 29, 2014; Accepted: October 08, 2014; Published: October 14, 2014

*Corresponding author: Mohammed Rahmatulla, Faculty of Life Sciences, University of Development Alternative, Dhanmondi, Dhaka-1209, Bangladesh, Tel: 017-1503-2621; Fax: 88-02-815-7339; E-mail: rahamatm@hotmail.com

\begin{abstract}
Toothache is a common problem throughout the world and can arise from causes like tooth decay and infected gums. While the former is treated with pain-relieving drugs, the latter may need treatment with antibiotics. Common pain-relieving drugs like Paracetamol, Aspirin and Diclofenac can produce adverse side-effects, while antibiotic use can lead to development of drug-resistant vectors, as well as adverse side-effects. Moreover, the majority of rural people of Bangladesh lack access to or cannot afford modern drugs and allopathic doctors. Medicinal plants can form an alternative, readily available and affordable way to treat toothache. In this review, we discuss the phytochemical constituents, antimicrobial and analgesic properties of a medicinal plant, Murraya paniculata, which has ethnomedicinal uses in the Indian sub-continent for treatment of toothache. We conclude that this plant can form an effective remedy for toothache treatment and merits scientific attention towards discovery of possibly novel drugs.
\end{abstract}

Keywords: Toothache, Murraya paniculata; Ethnomedicine; Antimicrobial; Analgesic

\section{Introduction}

Toothache is a common problem occurring in the human population throughout the world on a frequent basis. The causative factors behind toothache can be many, including tooth decay or fracture, abscessed tooth, or infected gums. Tooth pain can be excruciating, leading to disruptions of normal eating habits and life style, and if not treated properly can lead to loss of the tooth. Proper treatment of tooth pain requires visits to a qualified dentist, which is always not possible or affordable, particularly to the rural population of Bangladesh, and so for that matter the poorer segments or rural population of many under-developed countries.

Proper treatment of toothache involves primarily three factors: repairing or replacing the damaged tooth, treatment of tooth ache, and treatment of gum infections that may be primarily responsible for the tooth pain. While the first needs the attention of a dentist, the second and third factors may be fulfilled with Over-The-Counter (OTC) drugs like Aspirin, Paracetamol or Diclofenac and doctor-prescribed antibiotics. The various OTC pain-relieving drugs can produce adverse effects like gastric ulceration (Aspirin, Diclofenac) or hepatotoxicity (Paracetamol) from prolonged use or over-dosage, and it may be kept in mind that the rural people are mostly illiterate and so can accidentally take an over-dose of drugs with the mistaken belief of lessening the pain rapidly.

Rural people in Bangladesh depend heavily on folk medicinal system for treatment of diseases, in which system relies primarily on medicinal plants for treatment. It has been reported that one such medicinal plant is Murraya paniculata (L.) Jack, otherwise known as Murraya exotica L., belonging to the Rutaceae family. The folk medicinal practitioners of Jessore district in Bangladesh advise boiling the leaves of the plant in water and then gargling with the water (to which a little table salt has been added) three to four times daily for three days [1]. Stems of the plant are also used for toothache and oral care in India [2], where traditional practitioners advise brushing teeth with stems to get relief from toothache and for maintaining healthy gums and teeth. The objective of the present review is to detail the phytoconstituents of the plant, examine the reported antimicrobial and analgesic activities, and finally conclude with an account of the therapeutic potential of the plant in the treatment of toothache.

\section{Reported phytoconstituents}

A large number of phytoconstituents have been reported from the plant, which has been reviewed [3]. Coumarins isolated from flowers include scopoletine glycoside, scopoline, murralongin, merranzin hydrate, 5,7-dimethyl -8-(3'-methyl-2-ketobutyl) coumarin, murpanidine, auraptenol, 7-methoxy-8 (1'-ethoxy-2'hydroxyl-3'-methyl-3'-burenyl) coumarin, yuehgesins A-C, braylin, omphalocarpin, (-) murracarpine, murrayacarpines $\mathrm{A}$ and B; coumarins isolated from leaves include paniculatine, coumurrayin, osthol, 8-isopentenyllimettin, phebalosin, murralongin, imperatorin, murragin, isomexoticin, mupanidin, murpanicin, hainanmurpanin, isomerazin, 7-methoxy-8-(2'methyl-2'-formylpropyl) coumarin, murrayanone, murraculatin; coumarins isolated from roots include mexoticin, murrangatin, murralongin, murrangatine palmitate, sibiricin, omphamurin, murraol, murracarpin, murralonginol isovalerate, 
isomurralongial isovalerate, murrangatine isovalerate, chloculol, 6-methoxy-7-geranylloxy coumarin, umbelliferone, 8-(2'oxo-3'-methyl) butoxy-7-methoxy coumarin, minumicrolin; from fruits, scopoletine; and from stems, 7-(3-methyl-2butenyloxy)-8-(3-butenyl-3-methyl-2-oxo) coumarin, 7-0- $\beta$ D-glucopyranosylloxy-8-(3-butenyl-3-methyl-2-oxo) coumarin, 8-(butenyl-3'-methyl)-7-0- $\beta$-D-galactopyranoside, 7-methoxy-8(2'-isovaleryloxy-3-butenyl-3-methyl) coumarin, marmesin-4'0- $\alpha$-L-arabinopyranoside, 7-methoxy-8-(3-butenyl-3-methyl-2oxo) coumarin, and 7-methoxy-8-(butenyl-3'-methyl) coumarin [3]. Other reported coumarins include paniculacin from whole plant along with other compounds like umbelliferone, scopoletin, 4-hydroxybenzoic acid, trans-cinnamic acid, and $\beta$-sitosterol [4]. From the aerial parts, two new coumarins, namely murrmeranzin and murralonginal have been reported [5]. From the leaves, the coumarins 2'-O-ethylmurrangatin, murranganone and paniculatin have been reported [6].

Flavonoids isolated from fruits include 5,7,3',4',5'-pentamethoxy flavanol, 5,6,7,3', $4^{\prime}, 5^{\prime}$-hexamethoxyflavone, 3,5,6,7,3', 4', '5'-heptamethoxyflavone, $\quad 3,5,7,8,3^{\prime}, 4^{\prime}, 5^{\prime}$-heptamethoxyflavone, 3,5,7,8,3',4'-hexamethoxyflavone, 5-hydroxy3,7,8,3',4'-pentamethoxyflavone, 5-hydroxy-3,7,8,3',4',5'-hexamethoxyflavone (gardenin A), 8-hydroxy-3,5,7,3',4',5'-hexamethoxyflavone, and 5,7,3',4',5'-pentamethoxyflavanol; from leaves-gardenins A, C and E, exoticin, umhengerin, 5,3',5'-trihydroxy-6,7',4'-trimethoxyflavone, 5-hydroxy-6,7,8,3',4'-pentamethoxyflavone, 5,3'-dihydroxy-6,7,4',5'-tetramethoxyflavone, and 5,3',5'-trihydroxy-6,7,4'-trimethoxyflavone; from roots-methylN-methyl anthranilate; from stems-3,5,6,7,8,3',4',5'-octamethoxyflavone and $3,5,7,3^{\prime}, 4^{\prime}, 5^{\prime}$-hexamethoxyflavone. A complex mixture of 70 Polymethoxylated Flavonoids (PMFs) have been reported from leaves of the plant, which included 45 flavones, 17 flavanones or chalcones and 8 PMFs glycosides [7].

Alkaloids isolated from different parts of the plant include noracronycine, de-N-methylnocronycine, skimmianine, yuehchukene, paniculidines A-C, edulitine, murrapanine, murrayacarine, 3-formylindole, paniculol, and 3-prenylindole from roots; caffeine and murrayaculatine from fresh flowers; and tamynine from leaves [3].

\section{Antimicrobial activity}

Methanolic extract of leaves was found to be active against both Gram-positive and Gram-negative human pathogenic bacteria like Escherichia coli, Klebsiella pneumoniae, Salmonella typhi, Enterobacter faecalis, Pseudomonas aeruginosa, Shigella flexinerii, Staphylococcus aureus, and Shigella sonneii. Highest activity was demonstrated against $P$. aeruginosa. The antibacterial activity was attributed to presence of alkaloids, flavonoids, and phenolic compounds in the extract [8].

Aqueous extract of leaves reportedly showed antibacterial activity against multi-drug resistant human pathogens like $E$. coli, K. pneumoniae, and Proteus mirabilis [9]. A recent review has pointed out the plant's antibacterial efficacy against $K$. pneumoniae [10].

\section{Analgesic and anti-inflammatory activity}

Petroleum ether, ethyl acetate and methanol (equal proportions) extract of bark at doses of 200 and $400 \mathrm{mg}$ per $\mathrm{kg}$ reportedly reduced the frequency of acetic acid-induced writhing and prolonged the tail flicking latency in mice. However, the phytochemical constituent(s) behind these observed analgesic activities were not determined [11].

Ethanol extract of leaves showed considerable antinociceptive activity in acetic acid-induced writhing tests in mice at doses of 250 and $500 \mathrm{mg} / \mathrm{kg}$. At these two doses, the numbers of writhings were decreased, respectively, by 26.7 and $66.7 \%$. In comparison, a standard antinociceptive drug, diclofenac, when administered at a dose of $25 \mathrm{mg} / \mathrm{kg}$ decreased the number of writhings by $82.7 \%$, demonstrating that the leaves like the bark possesses phytoconstituents with significant pain-relieving potential [12].

The ethanol extract of leaves was tested for antinociceptive activity through hot plate and acetic acid-induced writhing tests in mice and anti-inflammatory activity through carragennan and dextran-induced rat paw edema and cotton pellets-induced granuloma in rats. At a dose of $200 \mathrm{mg} / \mathrm{kg}$, the extract reduced carrageenan-induced paw edema by $58.4 \%$, and dextran-induced paw edema by $54.4 \%$. At the same dose, cotton pellet-induced paw granuloma was reduced by $51.6 \%$ and acetic acid-induced writhings by $67.9 \%$ [13]. The results suggest that the leaves can be used to reduce gum inflammation and toothache, the latter occurring independently or in association with inflamed gums.

Methanolic extract of leaves also demonstrated antinociceptive activity in acetic acid-induced writhing tests and hot plate tests. An alkaloid, isolated from leaf extract, namely isomurrayafoline, was seen to be responsible for the observed effects [14]. Another compound, 2'-O-ethylmurrangatin, isolated from leaves of the plant reportedly inhibited lipooxygenase activity, and so can be a potential inhibitor of pain [15]. Ethanol extract of leaves showed antiinflammatory activity against carrageenin-induced paw edema in rats at doses of 300 and 600 $\mathrm{mg} / \mathrm{kg}$, which was comparable to that of aspirin at a dose of 150 $\mathrm{mg} / \mathrm{kg}$ at the highest dose tested [16].

\section{Therapeutic potential}

For treatment of toothache, two modes of treatment have been reported ethnomedicinally, either gargling with hot water extract of leaves [1] or brushing teeth with stems [2]. Therefore, relevant phytochemicals for relieving toothache must be searched among the leaves and stems. One such analgesic phytoconstituent, namely isomurrayafoline, has already been reported from leaves [14]. However, the plant contains many other phytoconstituents, which need to be scientifically studied for their analgesic and anti-inflammatory potentials.

Various flavonoids and alkaloids have been reported from leaves and aerial parts. These two groups of compounds have been implicated in numerous studies involving analgesic effects. For instance, phytochemical screening of Teucrium stocksianum methanolic extract demonstrating antinociceptive activity showed the presence of flavonoids among other groups of compounds [17]. The antiinflammatory effect of hydroalcoholic extract of Lampaya medicinalis has been attributed to presence of flavonoids in the extract [18]. The ameliorative potential of 
ethanolic extract of whole plant of Vernonia cinerea in the Chronic Constriction Injury (CCI) of sciatic nerve induced neuropathic pain in rats has also been attributed to flavonoids [19]. The antinociceptive and anti-inflammatory of flower extract of Alangium salvifolium has been attributed to presence of alkaloids and flavonoids in the extract [20]. Thus alkaloids and flavonoids present in leaves and stems of M. paniculata can account for its analgesic, anti-inflammatory, and possibly antimicrobial activities [8].

The mouth is inhabited by 200-300 bacterial species, but only a few of them can cause dental caries or tooth decay, leading to toothache. Streptoccus mutans is the main causative organism of tooth decay. It would be interesting to study antibacterial activity of leaf and stem of $M$. paniculata on $S$. mutans. Gum infections (gingivitis leading to periodontitis) can be caused by microorganisms like Actinobacillus actinomycetemcomitans, although other genera of bacteria have been implicated like Treponema, Bacteroides, Porphyromonas, Prevotella, Capnocytophaga, Peptostreptococcus, Fusobacterium, Actinobacillus, and Eikenella [21]. Further scientific work on the effect of $M$. paniculata whole plant or plant parts against these microbial genera and species can open up a new field in the treatment of tooth decay and gum diseases. But even without isolation of bioactive phytoconstituents, scientific validation of the pain relieving effect of $M$. paniculata in tooth of patients with tooth decay or gum infections can lead to its rational use by rural people of Bangladesh with confidence, and can also help adding crude extract of the leaves and stems of the plant in items like toothpaste, whose regular use can maintain proper oral hygiene and prevent tooth and gum diseases, besides alleviation toothache. Thus the plant merits further scientific attention from scientists towards discovery of possible novel antimicrobial and pain relieving compounds.

\section{References}

1. Islam F, Jahan FI, Seraj S, Malek I, Sadat AF, Bhuiyan MA, et al Variations in disease and medicinal plant selection among folk medicinal practitioners: a case study in Jessore district, Bangladesh. American-Eurasian Journal of Sustainable Agriculture. 2011; 5(2): 282-91.

2. Kumar RP. Ethno medicinal plants used for oral health care in India. International Journal of Herbal Medicine. 2014; 2(1): 81-7.

3. Gill NS, Kaur N, Arora R. An overview on: Murraya paniculata Linn. International Journal of Institutional Pharmacy and Life Sciences. 2014; 4(4): 1-11.

4. Saeed S, Shah S, Mehmood R, Malik A. Paniculacin, a new coumarin derivative from Murraya paniculata. J Asian Nat Prod Res. 2011; 13(8): 724-7. doi: 10.1080/10286020.2011.586343.

5. Saied S, Nizami SS, Anis I. Two new coumarins from Murraya paniculata. J Asian Nat Prod Res. 2008; 10(5-6): 515-9. doi: 10.1080/10286020801967292.

6. Choudhary MI, Azizuddin, Khalid A, Sultani SZ, Atta-ur-Rahman. A new coumarin from Murraya paniculata. Planta Med. 2002; 68(1): 81-3.

7. Zhang JY, Li N, Che YY, Zhang Y, Liang SX, Zhao MB, et al. Characterization of seventy polymethoxylated flavonoids (PMFs) in the leaves of Murraya paniculata by on-line high-performance liquid chromatography coupled to photodiode array detection and electrospray tandem mass spectrometry. J Pharm Biomed Anal. 2011; 56(5): 950-61. doi: 10.1016/j.jpba.2011.08.019.

8. Gautam MK, Gangwar M, Nath G, Rao CV, Goel RK. In-vitro antibacterial activity on human pathogens and total phenolic, flavonoid contents of Murraya paniculata Linn. leaves. Asian Pacific Journal of Tropical Biomedicine. 2012; S1660-3.

9. Chowdhury MAL, Ashrafuzzaman M, Ali MH, Liza LN, Zinnah KMA. Antimicrobial activity of some medicinal plants against multi drug resistant human pathogens. Advances in Bioscience and Bioengineering. 2013; 1(1): 1-24.

10. Shaik G, Sujatha N, Mehar SK. Medicinal plants as source of antibacterial agents to counter Klebsiella pneumoniae. Journal of Applied Pharmaceutical Science. 2014; 4(1): 135-47. doi: 10.7324/ JAPS.2014.40123.

11. Podder MK, Das BN, Saha A, Ahmed M. Analgesic activity of bark of Murraya paniculata. International Journal of Medicine and Medical Sciences. 2011; 3(4): 105-8.

12. Sharker SM, Shahid IJ, Hasanuzzaman M. Antinociceptive and bioactivity of leaves of Murraya paniculata (L.) Jack, Rutaceae. Revista Brasileira de Farmacognosia. 2009; 19(3): 746-8. doi:10.1590/S0102$695 X 2009000500016$.

13. Narkhede MB, Ajmire PV, Wagh AE. Evaluation of antinociceptive and anti-inflammatory activity of ethanol extract of Murraya paniculata leaves in experimental rodents. International Journal of Pharmacy and Pharmaceutical Sciences. 2012; 4(1): 247-50.

14. Fazal-ur-Rehman, Khan MF, Khan I, Shareef H, Marwat SK. Analgesic activity of carbazolealkaloid from Murraya paniculata Linn.(Rutaceae). American-Eurasian Journal of Agriculture \& Environmental Sciences. 2014; 14(3): 240-5.

15. Shaikh A, Choudhary MI. Bioassay studies of 2'-0-murrangatin isolated from a medicinal plant, Murraya paniculata. Turkish Journal of Biology. 2011; 35: 751-5.

16. Rahman MA, Hasanuzzaman M, Uddin N, Shahid IZ. Antidiarrhoeal and anti-inflammatory activities of Murraya paniculata (L.) Jack. Pharmacogyonline. 2010; 3: 768-76.

17.Shah SM, Sadiq A, Shah SM, Ullah F. Antioxidant, total phenolic contents and antinociceptive potential of Teucrium stocksianum methanolic extract in different animal models. BMC Complementary and Alternative Medicine. 2014; 14: 181. doi:10.1186/1472-6882-14181.

18. Morales G, Paredes A, Olivares A, Bravo J. Acute oral toxicity and anti-inflammatory activity of hydroalcoholic extract from Lampaya medicinalis Phil in rats. Biol Res. 2014; 47(1): 6. doi: 10.1186/07176287-47-6.

19. Thiagarajan VR, Shanmugam P, Krishnan UM, Muthuraman A. Ameliorative potential of Vernonia cinerea on chronic constriction injury of sciatic nerve induced neuropathic pain in rats. An Acad Bras Cienc. 2014; 86(3): 1435-50.

20.Zahan R, Nahar L, Nesa ML. Antinociceptive and anti-inflammatory activities of flower (Alangium salvifolium) extract. Pak J Biol Sci. 2013; 16(19): 1040-5.

21.Guthmiller JM, Novak KF. Periodontal Diseases. In: Polymicrobial Diseases, Chapter 8, Brogden KA, Guthmiller JM (Eds). Washington DC; ASM Press. 2002. 